\title{
An overview of a study on patients' experiences of bowel symptoms and symptom management strategies following surgery for rectal cancer
}

\author{
Margaret Landers
}

School of Nursing and Midwifery Brookfield Health Sciences Complex, UCC

\section{Background}

Colo-rectal cancer is reported as the second most common cancer among both males and females in Ireland with approximately 2,700 patients diagnosed annually. There has been a dramatic improvement in colo-rectal cancer survival over the last 15 years with approximately $50 \%$ of patients now expected to survive this cancer in Ireland National Cancer Registry (2009). Earlier diagnosis lends toward more effective and less invasive surgical treatments such as anal sphincter saving surgery. Sphincter saving surgery is now viewed as an advance over the traditional approach involving abdominal perineal surgery which typically leaves patients with a permanent colostomy for life. Sphincter saving surgery preserves the anal sphincter and therefore a permanent colostomy can be avoided.

A colostomy is a surgical procedure in which a stoma (or opening) is formed by bringing the end of the large intestine or colon through an incision in the abdominal wall and suturing it into place. A colostomy means that bowel motions are emptied from an opening in the abdomen into a (colostomy) bag. Although a permanent colostomy is avoided, bowel function may be altered following sphincter saving surgery. This is due to the fact that rectal reservoir capacity is changed after surgery which is caused primarily by a reduction in the size of the rectal remnant. As a consequence, sphincter saving procedures can lead to altered bowel symptoms both in the long and short term such as faecal incontinence, bowel urgency and bowel frequency.

A lack of published literature exists on the self-care strategies patients use to manage the bowel symptoms experienced following sphincter saving surgery. Moreover, it is not known if there is a relationship between the bowel symptoms experienced and the selfcare strategies patients use to manage symptoms, thereby presenting a gap in the literature. The lack of literature in this area may be due to the fact that bowel function is a very private matter for individuals and patients may be reluctant to talk openly about their bowel problems to researchers. However, many patients continue to live with poor bowel control following sphincter saving surgery which impacts on their daily life. Therefore, it 
is important that patients are given the opportunity to share their concerns about problematic bowel symptoms that they may be experiencing. It is also important to explore what self-care strategies patients find most helpful in managing their bowel symptoms.

\section{Data Collection}

For this study, a questionnaire was designed to collect data about patients' bowel symptoms experiences and symptom management strategies following sphincter saving surgery. Increased anonymity for patients is one of the most important factors in the use of questionnaires in a study. The embarrassing nature of the bowel symptoms experienced may make it difficult for some patients to discuss them at an interview. In addition, they might find it easier to write about their experiences in the comfort of their own home in the knowledge that their anonymity is maintained. The questionnaire asks patients to identify the bowel symptoms that they are currently experiencing and the self-care strategies that they find most effective in helping them to manage their symptoms. In addition, an open-ended question was included at the end of the questionnaire. The aim of this part of the questionnaire was to give patients the opportunity to write about the bowel symptoms that were most troublesome for them, in their own words. It was hoped that these comments would give further information on effects of the bowel symptoms on their everyday lives and the management strategies they used to deal with them.

\section{Findings}

Although the study is currently at data collection phase, some preliminary findings are available. Findings to date highlight that altered bowel motion is a common occurrence for patients following sphincter saving surgery for rectal cancer. The unpredictable nature of the bowel symptoms experienced following surgery caused some patients to fear losing control and becoming incontinent. A small number of patients are unsure what to expect in the long term following their surgery. In the open-ended question, one patient revealed that he had suffered from poor bowel control since the surgery and wondered if his bowel function would ever return to normal:

I often feel that my bowel symptoms will last for the rest of my life. (Participant 1)

It was noted that for the self-care strategies used in managing symptoms patients largely learned through trial and error. By adding certain foods to their diet, patients were able to treat symptoms such as diarrhoea and constipation. For example, some people developed strategies of excluding certain foods such as pulses or planning when and what food could be eaten. This involved determining which foods should be 'cut out' or 'put in'. However, the process of identifying offending foods was laborious and was not always successful for 
patients. In addition, medications were taken by some patients to manage symptoms with some degree of effectiveness. Patients also wore protective pads and used barrier creams and moist wipes to protect their skin from becoming sore.

Patients were constantly fearful of the risk of becoming incontinent. This was particularly the case in social situations, leisure activities and when at work. They were sensitive about the embarrassing nature of bowel symptoms experienced and the need to be adjacent to a toilet at all times. Some patients responded by avoiding, or withdrawing from social activities due to fear of experiencing an acute episode of faecal incontinence and the stress associated with not always having immediate access to a toilet. One patient reported that because his work involved travel, he could not go back to work as he was worried that he would not always have immediate access to a toilet. One patient who used public transport to get to work reported that he now:

avoids breakfast to allow for safe travel to work. (Participant 2)

However, the study also highlights that in some cases the bowel symptoms experienced following surgery improve over time. One patient reported that while he initially found the frequent visits to the toilet 'disheartening' his bowel symptoms had improved over time and as a result he now felt less anxious when out socially.

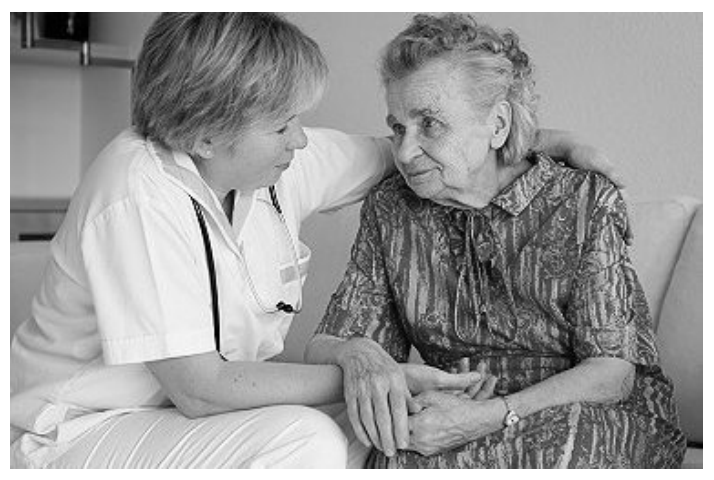

Two roads diverged in a wood and I... I took the one less travelled and it has made all the difference (Robert Frost)

Figure 1: Care of Patients

\section{Conclusion}

This study is giving patients the opportunity to share their experiences and views about the bowel symptoms they experienced following sphincter saving surgery for rectal cancer. This subject was largely unexplored in the literature. The study revealed that the bowel symptoms experienced following sphincter saving surgery caused both embarrassment and inconvenience to patients. The study also highlights the use of a variety of selfcare strategies by patients to manage symptoms, in an effort to prevent embarrassment 
and to live their lives as normally as possible. It is possible that the variety of self-care strategies adopted by patients to manage bowel symptoms may be unknown to health care providers but may be of interest to other patients. The findings from this study data will provide valuable information for health care professionals involved in the care of patients following sphincter-saving surgery for rectal cancer (Figure 1). It is anticipated that the findings will provide useful data to inform practice and future developments of interventions on self-care programmes, to help patients to manage their bowel symptoms both in the short term and long term following surgery.

Margaret Landers is a College Lecturer and a PhD Student at the School of Nursing and Midwifery Brookfield Health Sciences Complex. UCC Cork under the supervision of: Professor Eileen Savage School of Nursing and Midwifery Brookfield Health Sciences Complex, UCC, Professor Geraldine McCarthy Head of School of Nursing and Midwifery, Acting Head College of Medicine and Health Brookfield Health Sciences Complex, UCC. The author would also like to acknowledge funding through a Research Fellowship awarded by the Health Research Board. 